Article

\title{
Atomic-Force Microscopy Analyses on Dislocation in Extinction Bands of Poly(dodecamethylene terephthalate) Spherulites Solely Packed of Single- Crystal-Like Lamellae
}

\author{
Eamor M. Woo *, Graecia Lugito, Cheng-En Yang and Shih-Ming Chang \\ Department of Chemical Engineering, National Cheng Kung University, No. 1, University Road, \\ Tainan 701-01, Taiwan; graecia_lugito@yahoo.com (G.L.); inike9527@hotmail.com (C.-E.Y.); \\ jessamine38@gmail.com (S.-M.C.) \\ * Correspondence: emwoo@mail.ncku.edu.tw; Tel.: +886-6-275-7575 (ex. 62670) \\ Academic Editor: Helmut Cölfen
}

Received: 25 June 2017; Accepted: 8 September 2017; Published: 11 September 2017

\begin{abstract}
This study, using atomic-force and polarized-optical light (AFM and POM) microscopies on the extinction banded spherulites of poly(dodecamethylene terephthalate) (P12T) at high $T_{\mathcal{C}}=110{ }^{\circ} \mathrm{C}$ with a film thickness kept at 1-3 $\mu \mathrm{m}$, has verified that banded spherulites can be composed of stacks of entirely single-crystal-like lamellae free of any twisting, flipping, or bending, and no branching of lamellae. Defects in the crystal packing of extinction bands are present in both intra-band and inter-band regions. The intra-band defects originate from the miss-match in spiral-circling into circular bands while the inter-band defects are in the interfaces between successive bands where single crystals in the ridge are jammed to deformation, then suddenly precipitate prior to initiating another cycle of banding. The fish-scale lamellae, at the initiation of a cycle, are orderly packed as terrace-like single crystals; conversely, near or on the defected regions, they are highly jammed or squeezed and deformed to beyond recognition of their original single-crystal nature.
\end{abstract}

Keywords: polymer crystallization; lamellar assembly; poly(dodecamethylene terephthalate); dislocation; extinction bands

\section{Introduction}

Dislocations are a certain type of defect in the assembly of crystals. Though originating from metallurgy or small-molecule-compounds, the phenomena of crystal dislocations are also commonly seen in long-chain polymers with crystal packing. Dislocations in metallurgy are areas where the atoms are "out of position" in the crystal structure; similarly, in metals or small-molecule compounds, dislocations are generated and move when a stress is applied, and the motion of dislocations allows a slipped crystal deformation to occur. Such a definition and behavior can also be applied to the long-chain molecules in polymers. Price et al. [1-3] discussed dislocations in single crystals of polyethylene (PE), poly(oxymethylene), and poly(ethylene oxide) (PEO). Although widely known in general polymer single crystals, the dislocation defects have been much less discussed in the lamellae assembled into periodically ring-banded spherulites. For a long time, many investigators had treated the lamellae in ring-banded spherulites as perfectly and continuously twisted single-plate lamellae without defects; thus, the issues of possible defects or discontinuity in the "perfectly spiraling lamellae" had been ignored and left untouched. Keller et al. [4] and Lindenmeyer et al. [5] discussed the origins of dislocation in PE single crystals. An earlier study [6] also verified that poly(dodecamethylene terephthalate) (P12T, also named as PDoT), upon crystallization at high $T_{c}$ 's, can pack into periodically extinction banded spherulites where the narrow valley band is a sharply precipitated crevice but 
the wide ridge band is packed with numerous single crystalline lamellae aligned as roof-shingle structures (or fish-scale packing) along the circular circumferential direction of bands. Owing to the single-crystalline nature of the lamellae packed on the ridge bands of extinction-border banded spherulites, dislocation defects among the assembled lamellae are inevitable in the bands. This type of defect in the ring bands of spherulites, however, has evaded the attention of most investigators.

Banded patterns in spherulites are widely reported and studied for many polymers, and mechanisms for interpreting periodic bands and related phenomena have been long studied and widely debated. Nevertheless, the details of defects or distortion mechanisms in the periodic crystal assembly in the bands (including valleys vs. ridges) of banded spherulites have not been examined in due processes. Historically, most investigations have mainly approached the issues of periodic patterns in crystals from surface views; however, it should also be pointed out that the interior assembly may be masked by top-surface crystals. Furthermore, the crystals in spherulites of a normal film thickness $(1-30 \mu \mathrm{m})$ for polarized optical microscopy characterization cannot be simply assumed to be single-crystalline plates exhibiting monotonous spiraling without multiple branching or bending. Moreover, polymers are not the only category of materials that display such behavior. Crystals in non-polymers, such as low molecular weight $\left(\right.$ low- $\left.-\mathrm{M}_{\mathrm{W}}\right)$ organic and inorganic salts may exhibit such patterns under some circumstances [7-14]. That is, materials in nature that show crystal packing or aggregation may display periodic patterns under some circumstances that induce such an assembly. Thus, when analyzing banded patterns, one should take into account that low- $\mathrm{M}_{\mathrm{w}}$ compounds with no chain folding are also able to display banding behavior identical to those seen in polymers. Polymer crystals possess chain-folding in lamellae, which was the main reason that led investigators to believe in "chain-folding induced surface stress" being responsible for the "stress-induced twisting of lamellae". Nevertheless, one should be aware that if one surveys ring banding phenomena in nature away from polymer fields, many small-molecule compounds, with no chain-folding in forming crystals, can display similar banded patterns, which indicates that chain folding and its induced surface stress are not necessarily the only mechanisms responsible for the periodic assembly into circular rings in spherulites. In the fields of crystallography for small-molecule compounds, there are two common proposals for banded spherulites: helical crystals and rhythmic precipitation; yet there are also occasions that both mechanisms are simultaneously responsible for the banded patterns. For different materials, the banding patterns may look similar, but the surface fine morphologies may differ subtly or significantly. Nevertheless, away from the surface morphologies in thin films, the inner crystal structures of banded materials have very rarely been examined. As the surface topology may differ among different banding patterns, the inner structures may also differ correspondingly. Similar to the banded spherulites seen in solution-cast small-molecule compounds, some polymers, like isotactic polystyrene (i-PS) or poly(E-caprolactone) (PCL) [15-18], also exhibit extinction-banded spherulites upon casting from solutions in thin films. Apparently, the extinction banded (or "non-birefringent banded") spherulites are controlled and driven by kinetics in species diffusion vs. solvent evaporation rates and have nothing to do with chain-folding surface stresses as the phenomena are seen in long-chain polymers, as well as small molecules, with no chain folding in crystals.

Periodically banded patterns in spherulite crystals (or aggregated crystals) of all matters are common and widely reported in crystallography literature. They are characterized by periodic rings of different geometry (usually circular bands, often distorted into several variations from simple circles) with periodic variation in terms of optical birefringence. The successive bands can be of distinctly different optical properties and topology; but sometimes, the successive bands can also be of the same optical properties bordered with a discontinuity. As mentioned, regardless of the geometric shapes of repetitive crystal patterns, periodic ring bands in polymer spherulites are known to have two basic types in terms of optical birefringence: (1) bands with alternating opposite optical interference colors; and (2) bands with a sharp optical extinction border between them. The terminology differentiation among the optical variations of bands may be trivial, but mechanisms behind the formation of this type of repetitive packing patterns have yet to be fully probed, especially the less investigated bands with 
an optical extinction interface. The mechanisms of this repetition of bands appear to differ depending on the material systems or conditions of crystallization imposed during crystal growth. Although bands of opposite optical properties (contrast birefringence) have been more often investigated and reported in the literature, extinction-border bands of the same optical properties in successive bands have also been sporadically reported in some polymers, usually in spherulites crystallized by solvent evaporation. A previous study [6] has shown a detailed description of lamellae packing in peculiar P12T spherulites with extinction bands (either circular or petal-like) that are only formed at relatively high $T_{c}$ 's $\left(110-115^{\circ} \mathrm{C}\right)$. Depending on the optical birefringence patterns, the periodic bands in polymer spherulites can be classified into two major types: (I) bands with optical extinction borders, and (II) bands of alternating interference colors.

Some relevant background is worth discussing here to distinguish the major differences between the extinction bands and alternating-birefringence bands. One of the most studied types involves brightly opposite interference colors (blue/orange in tint plates) in successive bands, such as ring-banded spherulite in poly(ethylene adipate) (PEA), poly(butylene adipate) (PBA), poly(3-hydroxybutyrate) (PHB), and poly(trimethylene terephthalate) (PTT), etc., crystallized at respective suitable temperature ranges. The other type is comprised of the extinction-border (non-birefringent) banded spherulites, which is less studied, except for some classical reports on polyethylene (PE) spherulites [19-22], or earlier mentioned PCL and iPS upon solution evaporation [15-18]. In 2013, Nurkhamidah et al. [19] discovered that poly(L-lactic acid) (PLLA), at various film thicknesses $(300-5000 \mathrm{~nm})$ upon melt-crystallization at a specific $T_{c}$, can develop up-and-down extinction-banded spherulites, where all of the lamellae in bands are proven by AFM to be flat-on, individual lamellae with a thickness $=$ ca. $20 \mathrm{~nm}$, and no twisting. The very narrow "valley", i.e., the optical extinction as a border between bands, is actually a sharp crevice between two consecutive convex bands. The "extinction-border" bands are definitely different from the dual-color bands with alternating interference blue/orange colors (in tint plates).

As a matter of fact, there have been no reports in the literature regarding the crystalline morphology or banding behavior of P12T, either in bulk or thin-film states until a recent disclosure on the crystalline morphology of P12T crystallized at relatively high $T_{c}{ }^{\prime} \mathrm{s}$ [6]. This work further performed in-depth microscopic analyses to verify the mechanisms of packing and assembly mechanisms from single crystals to aggregated periodic bands. Owing to the single crystalline nature of lamellae assembly in the extinction bands, occasional crystal dislocations could be found. The aim of this work was to probe the mechanisms of these banding defects and gradient crystal assembly in the extinction bands that are packed of single-crystal-like lamellae.

\section{Experimental Section}

\subsection{Materials and Preparation}

Poly(dodecamethylene terephthalate) (P12T) was synthesized in house with a two-step polymerization from 1,12-dodecanediol and dimethyl terephthalate (DMT), with $0.1 \%$ butyl titanate as a catalyst [6]. The number-average molecular weight $\left(M_{n}\right)$ and the polydispersity index (PDI) as determined by gel permeation chromatography (GPC, Waters) were $9224 \mathrm{~g} \mathrm{~mol}^{-1}$ and 1.91, respectively. Melting and glass-transition temperatures of the synthesized P12T were determined with DSC to be $121.0^{\circ} \mathrm{C}$ and $-1.3{ }^{\circ} \mathrm{C}$, respectively.

A polymer solution was prepared with a properly weighed amount of purified P12T by dissolving in chloroform (1-2 wt \%), which was drip-cast or spin-cast (for different film thicknesses from 1-10 $\mu \mathrm{m}$ ) on glass slides for polarizing optical microscopy (POM), atomic-force microscopy (AFM), and scanning electron microscopy (SEM) observations. After solution casting and proper drying, the P12T cast films on glass were briefly melted at $165^{\circ} \mathrm{C}$ (for $1 \mathrm{~min}$ ), and then rapidly brought to an isothermal $T_{\mathcal{C}}=105-115^{\circ} \mathrm{C}$ for full crystallization $(\mathrm{t}=3-5 \mathrm{~min}$ ). Film thickness after subtracting the glass slide was estimated to be ca. $400-4000 \mathrm{~nm}$. For comparison, P12T films were also crystallized at lower $T_{c}=96^{\circ} \mathrm{C}$ 
for confirming the dramatic differences of optical patterns between spherulites crystallized at different $T_{c}$ 's. The optical differences suggest that the lamellae and their assembly therein the macro-spherulites are packed in different mechanisms driven by various factors.

\subsection{Apparatus}

Polarized-light optical microscopy (POM, Optiphot-2, Nikon, Tokyo, Japan), equipped with a Nikon Digital Sight (DS-U1, Nikon, Tokyo, Japan) digital camera and a hot stage (Linkam THMS-600, Linkam Scientific Instruments Ltd., Tadworth, UK) with a temperature programmer (T95, Linkam Scientific Instruments Ltd., Tadworth, UK), was used for characterizing the crystalline morphology of crystallized films, or monitoring the growth of spherulites. Atomic-force microscopy (AFM, diCaliber, Veeco Co., Santa Barbara, CA, USA) investigations were made in the intermittent tapping mode of AFM with a silicon tip ( $f=70 \mathrm{kHz}, r=10 \mathrm{~nm}$ ) installed. AFM measurements were also carried out to determine the height difference between the ridge and valley in P12T ring-banded samples. The top-surface morphology of crystallized samples was examined and characterized using scanning electron microscopy (SEM, Quanta-400F, FEI Company, Hillsboro, OR, USA) for revealing details of lamellar structures in banded spherulites.

\section{Results and Discussion}

Periodic extinction-banded spherulites (with either circular or flower-petal-like patterns), in dramatic contrast to the dual-birefringence ring-banded spherulites, are influenced by many kinetic factors during growth. In addition to $T_{c}$, both the film thickness and top confinement on P12T sample films imposed various extents of kinetics on spherulites growth, which led to different morphology patterns in the periodic extinction-banded spherulites. Numerous kinetic factors may influence the patterns and inter-band spacing of the extinction-banded P12T spherulites. It has been shown in a previous work [6] that ring bands with sharp optical extinction between successive bands in P12T only occur at relatively high $T_{c}$ 's such as $110^{\circ} \mathrm{C}$ (as well as a suitable range of film thickness), with dual types of spherulites co-existing at the same $T_{c}$. Such facts may reflect that a non-uniform thickness may be present across the sample films, leading to multiple spherulite patterns at the same $T_{c}$. By contrast, at lower $T_{\mathcal{c}}$ 's (such as $60-90^{\circ} \mathrm{C}$ ), no such types of extinction bands occur [6]; instead, circular bands of alternating blue/orange interference colors are present in lower- $T_{c}$-crystallized P12T spherulites. Petal-like extinction ring bands are present in uncovered thin $(<2 \mu \mathrm{m})$ P12T films; by contrast, for thicker P12T films (thickness $>5 \mu \mathrm{m}$ ), no such concentric bands are present in the spherulites (when crystallized at the same $T_{c}$ 's). For P12T films of any thickness, no such concentric or petal-like bands are present in the spherulites if the films are covered with a top-cover glass.

Figure 1 shows SEM micrographs for the morphology of the extinction-banded spherulites in the P12T film of 1-3 $\mu \mathrm{m}\left(\right.$ at $\left.T_{c}=110^{\circ} \mathrm{C}\right)$. Note that the fan-like dendrite crystals are growing in two opposite directions from the center, suggesting that the nuclei center is a region of complex and anisotropic structures; but the surrounding bands are more uniform in the lamellae structures. For brevity, not all SEM figures are shown, but the bottom of the figure shows four schemes of a collective SEM morphology and POM patterns that illustrate the effects of increasing film thickness on the banding patterns in P12T films (BOTTOM: from left to right: $400 \mathrm{~nm}$ to $5 \mu \mathrm{m}$ in thickness). The P12T films of all thickness ranges were crystallized at exactly the same $T_{C}=110{ }^{\circ} \mathrm{C}$ (all films uncovered during crystallization). Banding disappears in P12T spherulites of a film thickness greater than $5 \mu \mathrm{m}$ (where the average radius of spherulites ca. $10 \mu \mathrm{m}$ ), while extinction-bordered bands are obviously present, with the shape and banding space varying, in P12T films of a thickness from $400 \mathrm{~nm}$ to $4 \mu \mathrm{m}$. For banding to appear in the P12T spherulites, the radius of spherulites must be far greater than the spacing of bands $(20-30 \mu \mathrm{m})$. Kinetically, the extinction bands are driven by the periodic precipitation of crystals owing to the draining of species in the growth front; if the radius of the spherulite is smaller than what it takes for the crystals to precipitate, then there are no bands. 
This indicates that the film thickness determines the radius of spherulites, which in turn determines whether there would be periodic precipitation to form extinction bands in P12T spherulites.

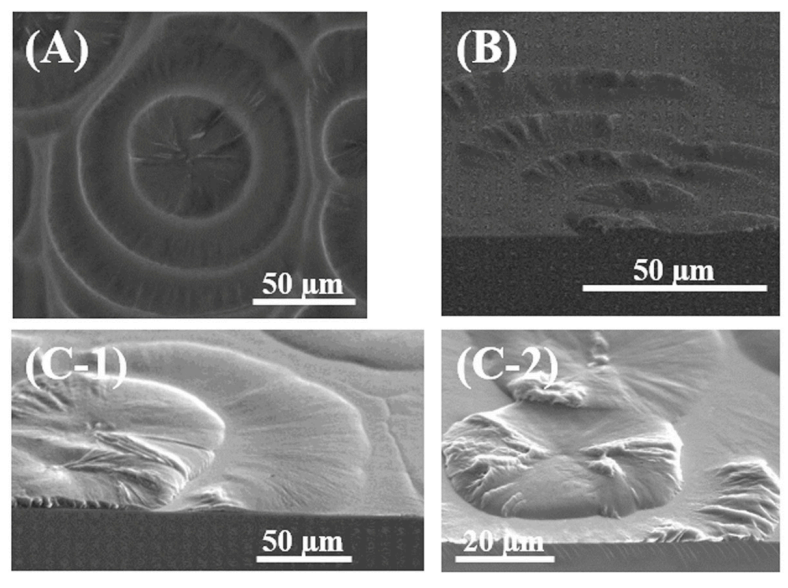

(D) Extinction Bands in P12T $\left(\mathrm{T}_{\mathrm{c}}=110^{\circ} \mathrm{C}\right)$

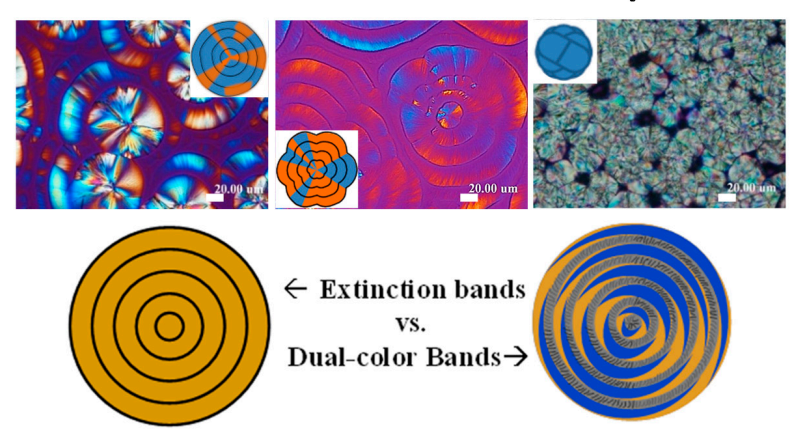

Figure 1. Effect of film thickness on the extinction-banded P12T spherulite patterns crystallized at $T_{C}=110^{\circ} \mathrm{C}$. SEM figures for periodically repetitive flower petals from nuclei with various film thicknesses of (A) $\sim 0.5 \mu \mathrm{m},(\mathbf{B}) \sim 1-2 \mu \mathrm{m},(\mathbf{C}-1, \mathbf{C}-2) 3.5 \mu \mathrm{m}$, from left to right (scale bars $=20 \mu \mathrm{m}$ ); and (D) schemes (from left to right) illustrating the variation of band patterns (black rings indicating a sharp extinction between bands) with increasing film thickness (400 nm-5 $\mu \mathrm{m})$. The POM image is reproduced with permission from ref. [6].

The effect of $T_{C}\left(100-115{ }^{\circ} \mathrm{C}\right)$ on the variation of band spacing of the extinction bands can be similarly assessed. For higher $T_{\mathcal{c}}$ values $\left(110-115^{\circ} \mathrm{C}\right)$, the species diffusion rates are higher (greater chain mobility) and growth can sustain a longer distance before coming to a periodic precipitation-leading to larger inter-band spacing. Vice versa, lower $T_{\mathcal{C}}$ values $\left(100-110{ }^{\circ} \mathrm{C}\right)$ lead to smaller inter-band spacing, as well as differences in banding patterns. Interestingly, the ring bands and their patterns in P12T spherulites crystallized at lower $T_{c}=60-96{ }^{\circ} \mathrm{C}$ are not influenced by the top cover on sample films (i.e., films sandwiched between the substrate and top cover glass). By contrast, at higher $T_{\mathcal{C}}$ values exceeding $100{ }^{\circ} \mathrm{C}\left(110-115^{\circ} \mathrm{C}\right)$, the banding characteristics of P12T are sensitively dependent on the cover or lack of cover on film samples during crystallization. Such facts indicate that the formation mechanisms and band characteristics of the extinction-banded spherulites at higher $T_{c}{ }^{\prime} \mathrm{s}$ $\left(T_{c}>100^{\circ} \mathrm{C}\right)$ may be significantly different from those of the P12T dual-birefringence-color banded spherulites crystallized at lower $T_{c}=60-96{ }^{\circ} \mathrm{C}$. Woo et al. [6] have reported AFM and POM graphs for regular defect-free extinction bands of $\mathrm{P} 12 \mathrm{~T}$, with no intra-band dislocation defects in P12T films of $1.5-2 \mu \mathrm{m}$ crystallized at $T_{c}=110^{\circ} \mathrm{C}$. The only periodic variation is noted in the inter-band extinction crevices across the entire P12T spherulites, where the optical extinction (see the POM graph insert) clearly corresponds to the periodic discontinuous crevices induced by the sharp vertical drop of crystals (according to AFM height profiles). 
Earlier corresponding AFM results on the fine morphology of the lamellar assembly clearly demonstrated that the P12T extinction-banded spherulites are all composed of flat-on lamellae in spherulites of various thin film thicknesses $(400 \mathrm{~nm} \sim 2 \mu \mathrm{m})$ crystallized at high $T_{c}=110^{\circ} \mathrm{C}$, with each of the lamella exhibiting a single-crystal-like geometry, stacked one on top of each other in a terrace-like structure (like the stacking in roof shingles) [6]. Thus, dislocations in the single-crystalline lamellae assembly could sporadically occur in the extinction P12T bands composed of single crystals. Periodic bands of various types in many polymers have been discussed; nevertheless, defects or dislocations in the bands are rarely analyzed. The focus of this work was placed on analyzing the origin and mechanisms of occurrence of occasional dislocations in the bands, which are shown in Figure 2 with AFM phase images and height profiles for P12T films crystallized at $T_{c}=110^{\circ} \mathrm{C}$. Figure $2 \mathrm{~A}$ shows the results (phase images and height profiles) of defect-free bands in P12T spherulites; while Figure 2B shows the counterpart results of bands with "intra-band" dislocation, which tend to be aligned in a straight line across the spherulites. In POM graphs, such defects are barely visible, usually as a sudden offset or smeared line cutting across the bands; by comparison, the AFM image in Figure 2 provides a much better-resolved view on the details of such defects than the POM images. The defect appears as a sudden convex arc as part of the band, which is also a slightly dislocated intersection of two portions of the circular/petal-like band to meet each other with an offset spacing. The offset is more significant for the inner bands; but becomes decreasingly less for the outer bands; with the offset spacing being $10 \mu \mathrm{m}$ for the inner bands and decreasing to $1 \mu \mathrm{m}$ for the outer ones [marked regions in AFM graphs]. The inter-band region is considered as a "defect" between the ring bands. The defect regions may be caused by crystals changing their direction all of a sudden, owing to the periodic draining/deficiency of available molten P12T chains for packing into crystals. Apparently, the results of height profiles on regular defect-free bands have revealed that the height variation is more orderly up-and-down, with a greater vertical drop of 750-1000 nm. By contrast, for the defect regions of the bands, the height profile shows a "flattened" variation of up-and-down with a much more depressed vertical drop of 100-300 nm. AFM zoom-in images of specific spots of the defect regions in the next figure will show more fine details for discussion. For further height profile analysis, Figure $2 \mathrm{C}$ shows height variation along the mid-part of the regions of defected bands (black line), and across the defected arc (blue line). One can clearly see that the dislocated region displays a significantly moreflattened profile in comparison to the regular banded regions. The height profile in the circumferential direction (blue line) shows that the region is much lower than the neighboring bands, forming a wide concave "U-shape". In the dislocated region, one can also see that the inner bands are offset to a much higher displacement than the outer bands. The much greater intra-band offset in the dislocation region makes the rings appear spiraling, rather than simple circular concentric, especially near the originating nuclei region (inner bands).
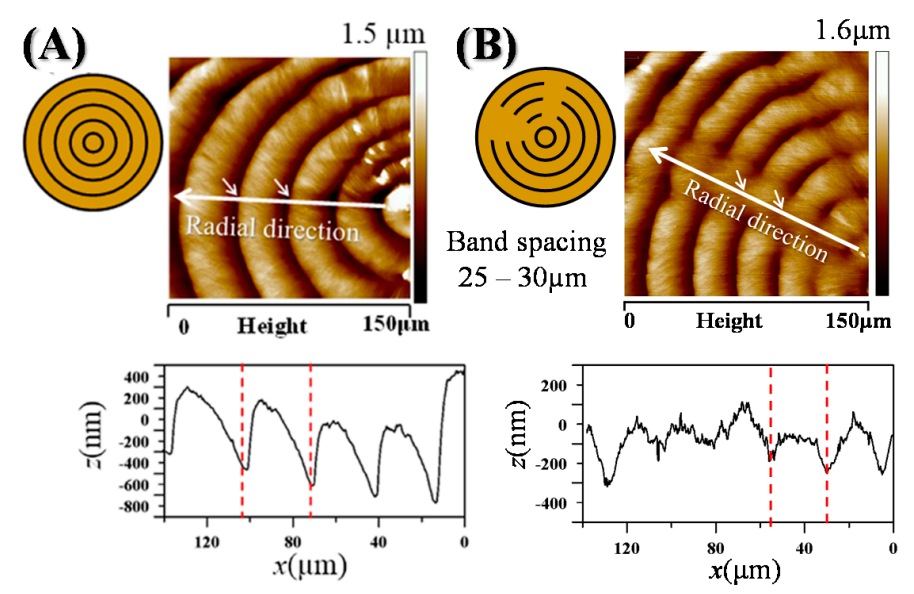

Figure 2. Cont. 
(C)

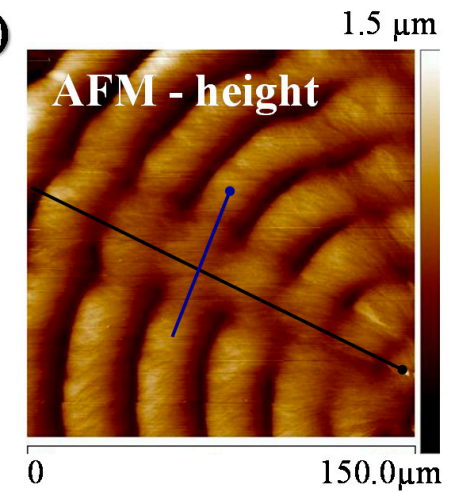

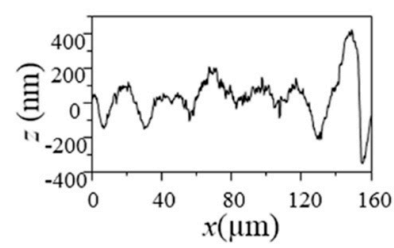

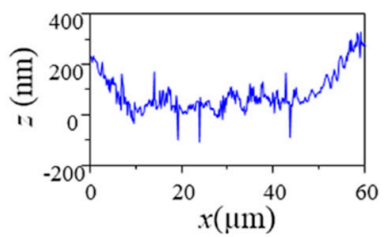

Figure 2. Comparison of AFM images and height profiles for (A) dislocation-free bands vs. (B) dislocated segments, (C) height profiles in the defect region in extinction ring bands of spherulites of P12T crystallized at $110{ }^{\circ} \mathrm{C}, 2 \mathrm{wt} \%$ (film thickness: $1.5-2 \mu \mathrm{m}$ ).

The dislocated offset regions of the band were further analyzed with AFM analysis. Figure 3 shows (Top) AFM 3D profiles and (bottom) respective zoom-in phase images of three regions (Spots-1,2,3) of the dislocated segments in the petal-like ring bands of $\mathrm{P} 12 \mathrm{~T}$ crystallized at $T_{c}=110{ }^{\circ} \mathrm{C}$. The respective phase images for these three regions are shown as zoom-in micrographs, with the numbers indicating three regions along the defect line of the band (inner band, intermediate band, and outer band). Region-1 is an inner band with a dislocation of greater offset $(8-10 \mu \mathrm{m})$. In the neighboring Region-1, the AFM image reveals that the regular band is composed of the orderly staking of single-crystalline lamellae assembly into a terrace-like structure; however, the offset in the dislocated spot shows completely smeared crystals of high irregularity. Region- 2 is a dislocation of medium offset $(4-5 \mu \mathrm{m})$. Similar features are seen in Region-1 and Region-2, except for the offset, which is lower in Region-2. By contrast, Region-3 comprises a dislocation of the smallest offset ( $1 \mu \mathrm{m}$ or less). In Region-3, the single-crystal assembly is much more orderly in the offset region, as well as in the neighboring regions of the outer band. However, as there is a concave cusp in the dislocation spot, the crystal assembly still shows some minor discontinuity, though not as much as that in Regions-1 or -2 .

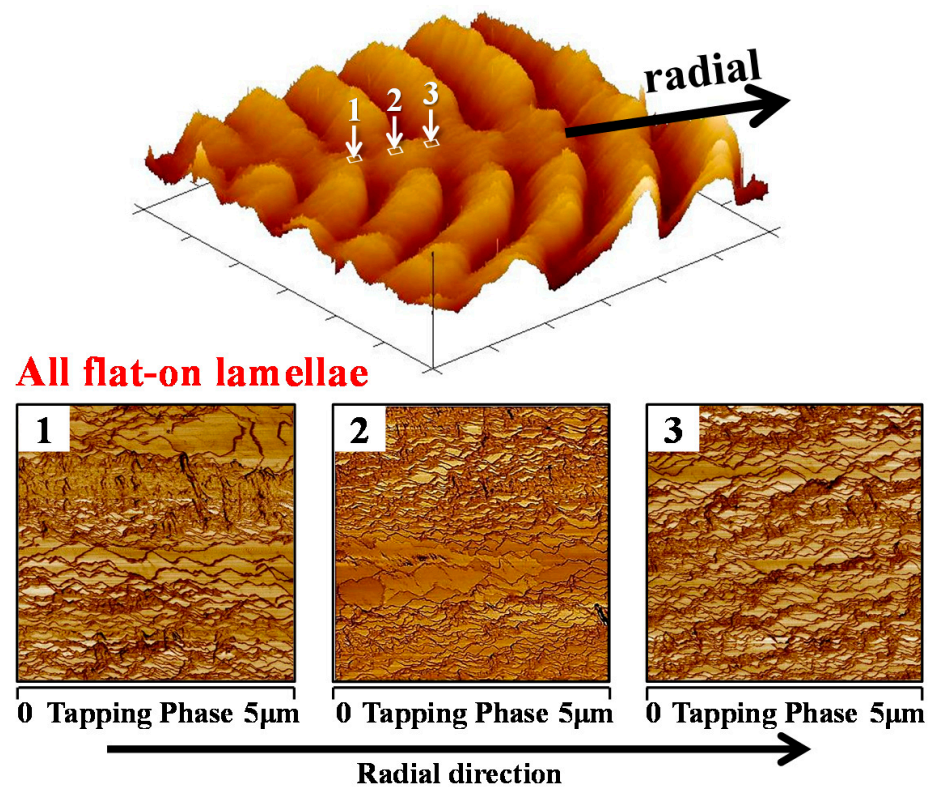

Figure 3. AFM 3D profiles and phase images on the dislocated segments in petal-like ring bands of $\mathrm{P} 12 \mathrm{~T}$ crystallized at $T_{C}=110^{\circ} \mathrm{C}$ (film thickness $\sim 1.5-2 \mu \mathrm{m}$ ). Spots-1,2,3 indicate three different regions of the dislocated defects for zoom-in analysis. 
Analysis of the defects is then directed to the upper left corner of the spherulites. The height profiles in the dislocated regions were compared to those of the regular bands. Height profiles along the dislocated bands, either radially across or circumferentially along the dislocated regions, were compared with the neighboring regular bands. The AFM height profile diagrams are illustrated in Figure 4, which shows the height image and analyses of height profiles of the regular bands with localized dislocations, along four regions of $a, b, c$, and d lines. Line-a (black-color line) indicates the height profile radially across the regular bands, showing periodic height variation of ca. $200 \mathrm{~nm}$. Line-b (blue-color line) shows a flattened height profile variation of ca. $100 \mathrm{~nm}$. Line-c (red-color line) shows that the bands are much much compressed, showing a narrower periodicity of ca. $3 \mu \mathrm{m}$ in comparison to that of the regular band $(5 \mu \mathrm{m})$. Line-d (green-color line) shows the height profile in the circumferential direction of regular bands, which are flat and not U-shaped, other than localized minor variations of height profiles owing to single-crystalline lamellae assembled as roof-shingle packing.

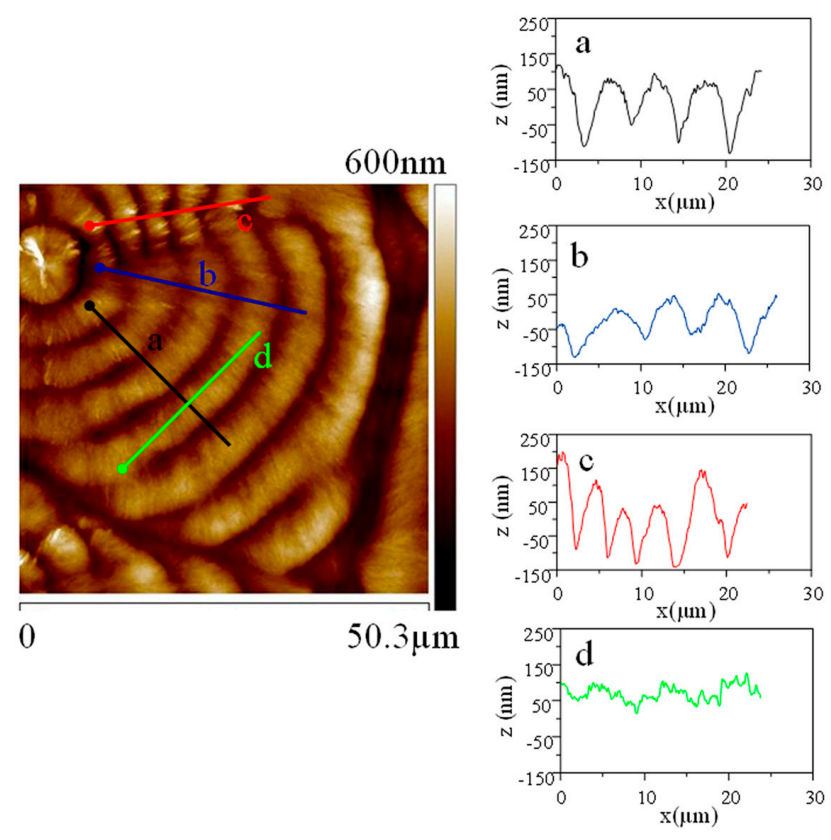

Figure 4. AFM height image (left) and profiles along the lines (a-d) showing regular bands with localized dislocations. Film thickness $=1.5-2.0 \mu \mathrm{m}$. AFM image is reproduced with permission from ref. [6].

For finer and more advanced analyses, the intra-band dislocation (defects in the bands packed of single-crystalline lamellae) is compared to the inter-band crevice region, where one band precipitates to near the substrate while the neighboring band just begins to rise. Figure 5 shows AFM analyses (phase images and height profiling across successive bands) on the inter-band regions of P12T spherulites crystallized at $T_{\mathcal{c}}=110^{\circ} \mathrm{C}$. Figure $5 \mathrm{~B}$ shows that in the initial growth cycle, lamellae are orderly packed as fish-scale terraces, or series-staggered terraces, with height profiles across individual single crystals (lozenge-shape lamellae) being ca 10-20 $\mathrm{nm}$. The nature of the single-crystalline lamellae packing in the extinction-banded P12T spherulites is clearly evident if one carefully examines the dimensions of the fish-scale structure as revealed in the zoomed-in AFM images, at the initial height-rising region of the bands. For comparison, Figure $5 \mathrm{~A}$ shows that the inter-band region is considered as a "defect" between the ring bands, where the lamellae are highly jammed and distorted and precipitate suddenly into a crevice of a $260 \mathrm{~nm}$ vertical drop in height. The defect regions may be caused by crystals precipitating downward and changing their direction all of a sudden, owing to the periodic draining/deficiency of available molten P12T chains for packing into crystals. The growth of lamellae in spherulites is thus not continuous but interrupted intermittently, with the interruption period depending on the $T_{\mathcal{C}}$, film thickness, top cover glass, etc., until the impingement of spherulites, leading 
to extinction-banded spherulites. The intra-band defect, instead, may be caused by screw-dislocation growth, where crystals change their orientations. In the regular dislocation-free bands, the crystal packing is a monotonously single crystalline with a shingle-like (fish-scale) structure, with the tip of each of the lozenge-shaped crystals pointing in a circumferential direction (perpendicular to the radial growth direction). As discussed earlier, within an extinction band, three localized regions with different morphologies can normally be divided by a band across their band width; however, for this ultra-thin film (spin cast to ca. $1000 \mathrm{~nm}$ ), the differentiation among the three regions is not significant in comparison to thicker films, to be shown and discussed later.

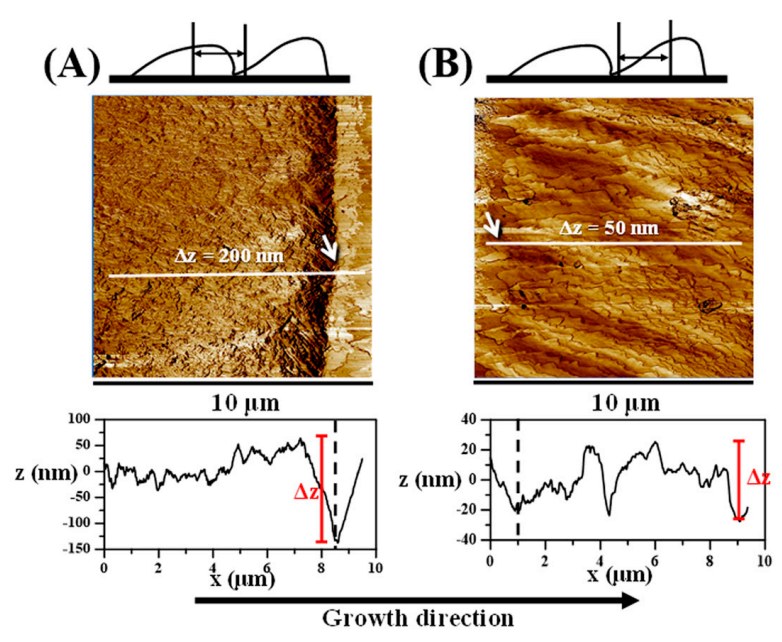

Figure 5. AFM analyses on the inter-band regions: (A) gradual rise at the beginning of the growth cycle of the next band; (B) precipitation at the end of the growth cycle into a crevice ( $260 \mathrm{~nm}$ in vertical depth) (film thickness $=$ ca. $1 \mu \mathrm{m})$.

Note that in this relatively thin P12T film (ca. $1 \mu \mathrm{m}$ ), each lamella is clearly visible; however, it is hard to tell the width of the lamellae as they overlap in their roots and also laterally overlap with each other. The thickness of lamellae is estimated to be tens of nanometers, which agrees with the dimensions of most polymer single crystals. All fish-scale-like lamellae have zig-zag peripheries, suggesting some local instability in growth. In the terrace-like packing, the lamellae partially overlap in their roots, with the crystal order pointing perpendicularly to the radial direction; thus, only one side of each of the lamellae is exposed. The lamellae crystals of single-side exposure with root-overlapping are thus assembled in patterns resembling the packing of fish-scales. For this thin P12T film, the geometry differences between the lamellae of the initial growth vs. end region may not be very significant. However, by comparing the initial growth region and end region of the band, one can still see differences within the band in that the lamellae overlap more loosely in the initial growth, while the lamellae overlap in their roots much more closely/compactly in the end region. In the inter-band region, there are clear interfaces signaling an intersection of two different crystal species of different orientations (precipitating downward vs. gradually upward). To sum up, for these thin P12T films, the vertical drop in the inter-band crevices is slight in comparison to thicker P12T films (2 or $3 \mu \mathrm{m})$, and thus, the distortion of the crystals' geometry/orientation is less. For thicker P12T films $(3.5-4 \mu \mathrm{m})$, to be discussed in later sections, the contrasts and differences among the different growing regions are much more significant.

In an earlier investigation of P12T [6], periodic evolution of lamellar morphology along three growth regions within a band was assessed on a thin-film P12T spherulite (thickness $=1-2 \mu \mathrm{m}$ ). For the thicker film, the vertical drop from peak to valley was found to be more significantly different in terms of geometry. AFM analysis was similarly performed on a slightly thicker P12T film $(3.5 \mu \mathrm{m})$. Figure 6 shows AFM 3D profiles and zoom-in phase images of three regions in petal-like bands of the P12T film crystallized at $T_{c}=110{ }^{\circ} \mathrm{C}$ (film thickness $\sim 3.5 \mu \mathrm{m}$ ). Three regions of growth are 
marked for zoom-in analyses with AFM phase imaging: (1) beginning of an emerging band, (2) peak maximum, and (3) precipitation near the valley. Generally, the variation trend of lamellar morphology across the band is similar among P12T films of different thicknesses (1-2 $\mu \mathrm{m}$ vs. $3.5 \mu \mathrm{m})$. The initial growth is typified with larger and more widely-spaced single-crystalline lamellae, whose packing is increasingly strained into crowdedness as the band is packed into the peak maximum. However, for the thicker P12T films $(\sim 3.5 \mu \mathrm{m})$ in this work, the differences in the lamellae morphology of the three regions $(1,2,3)$ of the band are more significant than those in thinner films. In Region-1, one can see that each of the lamellae is at least $3 \mu \mathrm{m}$ in width and ca. tens of nanometers in lamellar thickness. In these phase images, the white arrow indicates the radial direction. The tips of the lamellae are zig-zag shaped in the lamellar peripheries, suggesting some local instability in growth. The lamellae partially overlap in their roots but emerge as distinct single-crystal lamellae, resembling the packing of fish-scales. In region-2 (peak), the dimensions of the lamellae are much smaller, being ca. $1 \mu \mathrm{m}$ in width, and there is a higher frequency of overlapping among the successive lamellae, indicating greater crowdedness. In Region-3 (crystal precipitation to valley), none of the crystals could be associated with single-crystalline dimensions, owing to significant jamming, overlapping, and perhaps deformation of the lamellae. It should be noted here, owing to a significant height drop in this Region-3, AFM characterization was also restricted, leading to a not so well resolved image of aggregated crystals. All crystals in Region-3 are jammed or deformed, with a morphology similar to the intra-crack dislocation defects discussed earlier (refer to Figure 3). Thus, the inter-band crystal precipitation and intra-band crystal dislocation likely share some similar origins in that they contain crystals of highly distorted geometries that are jammed or suddenly screwed into different directions from the original growth. Interestingly, note the dramatic contrast between the crystals of precipitation and initial growth in Region-3. The upper right corner of Region-3 clearly shows the beginning growth of a successive band, where single-crystal lamellae of large dimensions can be easily discerned, in dramatic contrast to the highly distorted crystals in the precipitated valley shown on the diagonal dark zone in the lower left of the AFM phase image. One sees that the gradual transition of morphologies from terrace-like wide lozenge single-crystal lamellae to highly jammed crystals, eventually leading to a vertical drop to near the substrate surface within one band of the $3.5 \mu \mathrm{m}$ thick $\mathrm{P} 12 \mathrm{~T}$ film, is quite similar to that in P12T films of other thicknesses. The aggregation and jamming of crystals near the top of the band (Regions-2 and 3) in thicker P12T films are much more pronounced than in the thinner films.

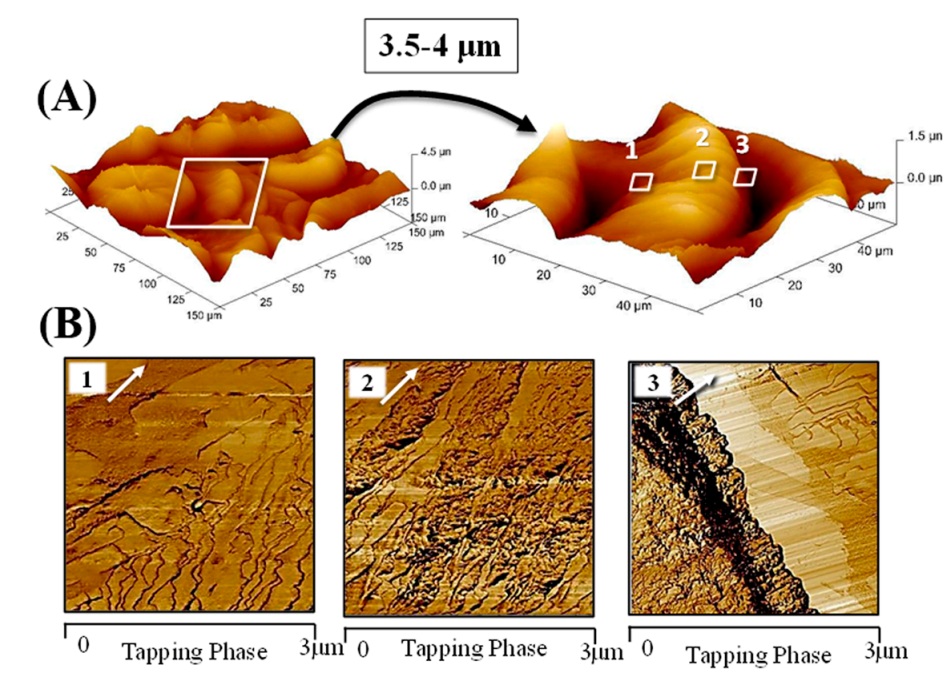

Figure 6. AFM 3D profiles (A) and zoom-in phase images $(B)$ of three regions in petal-like bands of the P12T thicker film crystallized at $T_{c}=110{ }^{\circ} \mathrm{C}$ (film thickness $\sim 3.5 \mu \mathrm{m}$ ). Regions-1,2,3 correspond to initial packing, peak, and inter-band discontinuity, respectively. White arrows in images indicate the radial direction. 
Inter-band precipitation can also be considered as a defect in crystal packing. The "inter-band" region is the interface between two successive concentric bands, where the growth cycle occurs again to initiate a new band after precipitation. Figure 7 shows AFM phase images and height profiles along the corresponding white line on inter-band defects in extinction bands of P12T thin films of two thickness levels: (A) $1.5 \mu \mathrm{m}$ and (B) $3.5 \mu \mathrm{m}$. The terrace-like single-crystal lamellae are packed as a fish-scale structure at the beginning of the banding cycle, prior to precipitating into a valley that is almost empty and near the substrate glass surface. The empty valley (free of discernible crystals) is responsible for the sharp/narrow optical extinction. Near the inter-band interface, one side (the end of maximum ridge going to precipitate) is highly jammed with distorted single-crystal lamellae, while the opposite side of the interface (the empty valley about to initiate another cycle) is smooth and free of visible crystals. At the beginning of the banding cycle, the terrace-like lamellae display 10-20 nm height variations $(\Delta z)$. At the end of the banding cycle, however, the vertical precipitation height ranges across two successive bands are found to be $450 \mathrm{~nm}$ (for the $1.5 \mu \mathrm{m}$ sample) and $1000 \mathrm{~nm}$ (for the $3.5 \mu \mathrm{m}$ sample). The dashed lines on the profile images indicate the position of the empty valley of the two samples.

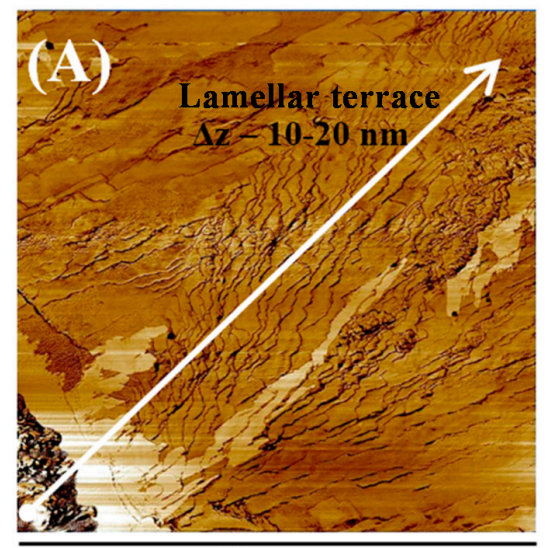

$10 \mu \mathrm{m}$

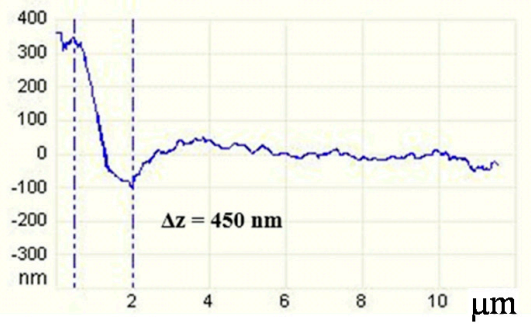

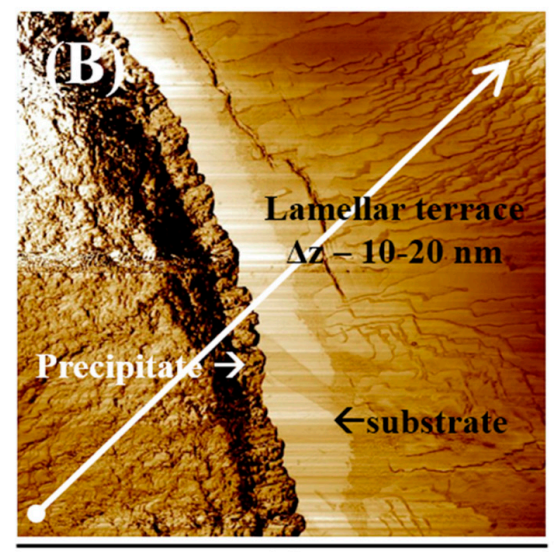

$10 \mu \mathrm{m}$

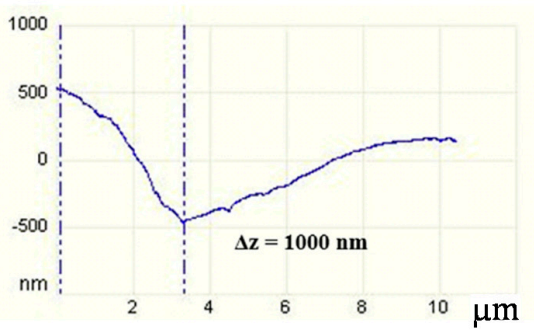

Figure 7. AFM phase images and height profiles on inter-band defects in extinction bands of the P12T thin film crystallized at $T_{\mathcal{C}}=110^{\circ} \mathrm{C}$, film thickness: (A) $\sim 1.5$ and (B) $3.5 \mu \mathrm{m}$, respectively.

\section{Conclusions}

This study, using AFM and POM microscopes for investigating the extinction banded spherulites of P12T at high $T_{c}=110^{\circ} \mathrm{C}$ with film thicknesses of $1-3 \mu \mathrm{m}$, has proved that banded spherulites can be composed of entirely single crystals free of any twisting, flipping, or bending, and no branching of lamellae. The single-crystal lamellae in the extinction banded P12T spherulites are all packed flat-on in a terrace-like fish-scale structure. In addition, the successive bands are not continuous, but are characterized by a distinct discontinuity, which is the inter-band precipitation of lamellae into a narrow valley free of any crystals near the substrate surface. The empty valley (free of discernible crystals) is responsible for the sharp/narrow optical extinction as viewed in the POM graphs. Such extinction-banded P12T spherulites are different in terms of their general morphology, lamellar 
assembly, and optical birefringence when compared to the birefringent-banded spherulites with alternating blue/orange crystal interference colors.

Defects in crystal packing are present in the intra-band and inter-band regions. Intra-band defects originate from the miss-match in spiral-circling into circular bands. Inter-band defects are in the interfaces between successive bands where single crystals in the ridge are jammed to deformation, then suddenly precipitate prior to initiating another cycle of banding. The fish-scale lamellae packing near or on the defected regions are jammed, deformed, or squeezed to beyond recognition of its original single-crystal nature.

Acknowledgments: This work has been financially supported by a basic research grant (MOST 105-2221-E-006246-MY3) for three consecutive years from Ministry of Science and Technology (MOST), to which the authors express their gratitude.

Author Contributions: This article was written through the contributions of all authors. All authors have given approval to the final version of the manuscript. Eamor M. Woo (professor at NCKU) conceived the original ideas, guided the work, analyzed the data, and wrote the manuscript with the help of Graecia Lugito (postdoctoral research fellow at NCKU), and Cheng-En Yang and Shih-Ming Chang (graduate master-degree students at NCKU), who conducted the experiments and collected the data.

Conflicts of Interest: The authors declare no conflict of interest. The founding sponsors had no role in the design of the study; in the collection, analyses, or interpretation of data; in the writing of the manuscript, and in the decision to publish the results.

\section{References}

1. Barnes, W.J.; Price, F.P. Morphology of polymer crystals: Screw dislocations in polyethylene, polymethylene oxide and polyethylene oxide. Polymer 1964, 5, 283-292. [CrossRef]

2. Barnes, W.J.; Luetzel, W.G.; Price, F.P. Crystallization of poly-(ethylene oxide) in bulk. J. Phys. Chem. 1961, 65, 1742-1748. [CrossRef]

3. Price, F.P. Markoff chain model for growth of polymer single crystals. J. Chem. Phys. 1961, 35, 1884. [CrossRef]

4. Mitsuhashi, S.; Keller, A. The morphology of multilayer polymer crystals. Polymer 1961, 2, 109-112. [CrossRef]

5. Holland, V.F.; Lindenmeyer, P.H. Morphology and crystal growth rate of polyethylene crystalline complexes. J. Polym. Sci. 1962, 57, 589-608. [CrossRef]

6. Lugito, G.; Woo, E.M.; Chang, S.M. Periodic extinction bands composed of all flat-on lamellae in poly(dodecamethylene terephthalate) thin films crystallized at high temperatures. J. Polym. Sci. Part B Polym. Phys. 2017, 55, 601-611. [CrossRef]

7. Shtukenberg, A.G.; Freudenthal, J.; Kahr, B. reversible twisting during helical hippuric acid crystal growth. J. Am. Chem. Soc. 2010, 132, 9341-9349. [CrossRef] [PubMed]

8. Shtukenberg, A.G.; Cui, X.; Freudenthal, J.; Gunn, E.; Camp, E.; Kahr, B. Twisted mannitol crystals establish homologous growth mechanisms for high-polymer and small-molecule ring-banded spherulites. J. Am. Chem. Soc. 2012, 134, 6354-6364. [CrossRef] [PubMed]

9. Gunn, E.; Sours, R.; Benedict, J.B.; Kaminsky, W.; Kahr, B. Mesoscale chiroptics of rhythmic precipitates. J. Am. Chem. Soc. 2006, 128, 14234-14235. [CrossRef] [PubMed]

10. Cui, X.; Rohl, A.L.; Shtukenberg, A.; Kahr, B. Twisted aspirin crystals. J. Am. Chem. Soc. 2013, 135, 3395-3398. [CrossRef] [PubMed]

11. Shtukenberg, A.; Freundenthal, J.; Gunn, E.; Yu, L.; Kahr, B. Glass-crystal growth mode for testosterone propionate. Cryst. Growth Des. 2011, 11, 4458-4462. [CrossRef]

12. Imai, H.; Oaki, Y. Emergence of helical morphologies with crystals: Twisted growth under diffusion-limited conditions and chirality control with molecular recognition. CrystEngComm 2010, 12, 1679-1687. [CrossRef]

13. Shtukenberg, A.; Gunn, E.; Gazzano, M.; Freudenthal, J.; Camp, E.; Sours, R.; Rosseeva, E.; Kahr, B. Bernauer's Bands. ChemPhysChem 2011, 12, 1558-1571. [CrossRef] [PubMed]

14. Oaki, Y.; Imai, H. Amplification of chirality from molecules into morphology of crystals through molecular recognition. J. Am. Chem. Soc. 2004, 126, 9271-9275. [CrossRef] [PubMed]

15. Duan, Y.; Jiang, Y.; Jiang, S.; Li, L.; Yan, S.; Schultz, J.M. Depletion-induced nonbirefringent banding in thin isotactic polystyrene thin films. Macromolecules 2004, 37, 9283-9286. [CrossRef] 
16. Duan, Y.; Zhang, Y.; Yan, S.; Schultz, J.M. In situ AFM study of the growth of banded hedritic structures in thin films of isotactic polystyrene. Polymer 2005, 46, 9015-9021. [CrossRef]

17. Wang, Z.; Hu, Z.; Chen, Y.; Gong, Y.; Huang, H.; He, T. Rhythmic growth-induced concentric ring-banded structures in poly ( $\varepsilon$-caprolactone) solution-casting films obtained at the slow solvent evaporation rate. Macromolecules 2007, 40, 4381-4385. [CrossRef]

18. Wang, Z.; Alfonso, G.C.; Hu, Z.; Zhang, J.; He, T. Rhythmic growth-induced ring-banded spherulites with radial periodic variation of thicknesses grown from poly( $\varepsilon$-caprolactone) solution with constant concentration. Macromolecules 2008, 41, 7584-7595. [CrossRef]

19. Nurkhamidah, S.; Woo, E.M. Unconventional non-birefringent or birefringent concentric ring-banded spherulites in poly(l-lactic acid) thin films. Macromol. Chem. Phys. 2013, 214, 673-680. [CrossRef]

20. Keller, A. Investigations on banded spherulites. J. Polym. Sci. 1959, 39, 151-173. [CrossRef]

21. Keith, H.D.; Padden, F.J. Banding in polyethylene and other spherulites. Macromolecules 1996, $29,7776-7786$. [CrossRef]

22. Bassett, D.C.; Hodge, A.M. On lamellar organization in banded spherulites of polyethylene. Polymer 1978, 19, 469-472. [CrossRef]

(C) 2017 by the authors. Licensee MDPI, Basel, Switzerland. This article is an open access article distributed under the terms and conditions of the Creative Commons Attribution (CC BY) license (http://creativecommons.org/licenses/by/4.0/). 